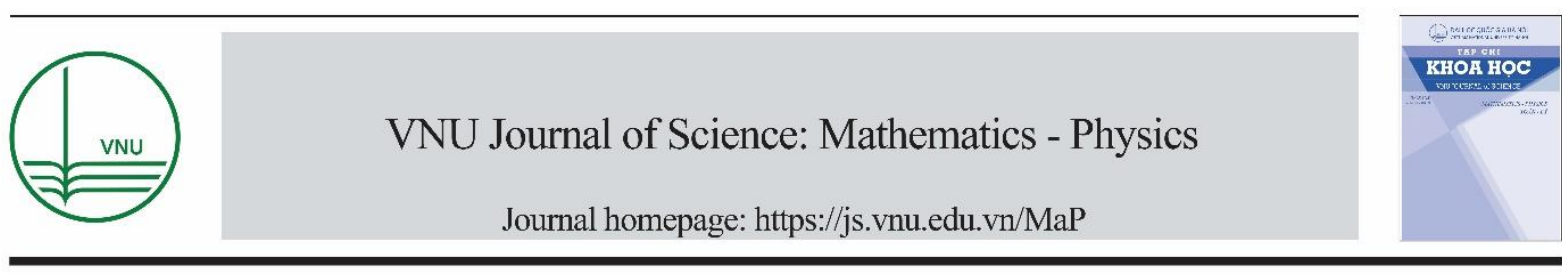

\title{
Convergence for Martingale Sequences of Random Bounded Linear Operators
}

\author{
Tran Manh Cuong ${ }^{1, *}$, Ta Cong Son ${ }^{1}$, Le Thi Oanh ${ }^{2}$ \\ ${ }^{I}$ Faculty of Mathematics, Mechanics and Informatics, VNU Hanoi University of Science, \\ 334 Nguyen Trai, Hanoi, Vietnam \\ ${ }^{2}$ Department of Mathematics, Hong Duc University, 565 Quang Trung, Dong Ve, Thanh Hoa, Vietnam
}

Received 03 December 2018

Revised 20 December 2018; Accepted 20 December 2018

\begin{abstract}
In this paper, we study the convergence for martingale sequences of random bounded linear operators. The condition for the existence of such a infinite product of random bounded linear operators is established.

AMS Subject classification 2000: 60H05, 60B11, 60G57, 60K37, 37L55.

Keywords and phrases: Random bounded linear operators, products of random bounded linear operators, martingales of random bounded linear operators, convergence of random bounded linear operators.
\end{abstract}

\section{Introduction}

Let $(\Omega, \mathcal{F}, P)$ be a complete probability space and $\mathrm{X}, \mathrm{Y}$ be separable Banach spaces. A mapping $\Phi: \mathrm{X} \rightarrow \mathrm{L}_{0}^{Y}(\Omega)$ is said to be a random operator, where $\mathrm{L}_{0}^{Y}(\Omega)$ stands for the space of $Y$. valued random variables and is equipped with the topology of convergence in probability. If a random $\Phi: \mathrm{X} \rightarrow \mathrm{L}_{0}^{Y}(\Omega)$ is linear and continuous then it is called a random linear operator. The set of all random linear operators $\mathrm{A}: \mathrm{X} \rightarrow \mathrm{L}_{0}^{Y}(\Omega)$ is denoted by $L(\Omega, \mathrm{X}, \mathrm{Y})$.

The random operator theory is one of the branches of the theory of random processes and functions; its creation is a natural step in the development of random analysis. Research in theory of random operators has been carried out in many directions such as random fixed points of random operators, random operator equations, random linear operators (see [1-4]).

\footnotetext{
${ }^{*}$ Corresponding author. Tel.: 84-912589676.

Email: cuongtm@vnu.edu.vn

https//doi.org/ 10.25073/2588-1124/vnumap.4305
} 
Martingale limit theorems are studied by many authors in recent years (see, e.g. [5-8] and references therein).

The study of multiplicative limit theorems was initiated by Bellman who considered the asymptotic behavior of the product

$$
A_{n}(\omega)=X_{n}(\omega) X_{n-1}(\omega) \ldots X_{1}(\omega)
$$

where $\left(\boldsymbol{X}_{\boldsymbol{k}}\right)$ is a stationary sequence of $\boldsymbol{k} \times \boldsymbol{k}$ random matrices. Belman showed that if $\left(\boldsymbol{X}_{\boldsymbol{k}}\right)$ are independent and have strictly positive elements then under certain conditions, a weak multiplicative law of large numbers exists a.s. The study of the asymptotic behavior of the products of random matrices is of very importance in the analysis of the limiting behavior of solutions of systems of differential and difference equations with random coefficients (see [9] and references therein). Recently, Thang and Son [2] obtained the convergence of the products of random linear operators $\left\{\boldsymbol{U}_{n}\right\}$ and $\left\{\boldsymbol{V}_{\boldsymbol{n}}\right\}$ of the form

$$
\begin{aligned}
U_{n}=\left(I+A_{n}\right)\left(I+A_{n-1}\right) & \ldots\left(I+A_{2}\right)\left(I+A_{1}\right), \\
V_{n} & =\left(I+A_{1}\right)\left(I+A_{2}\right) \ldots\left(I+A_{n-1}\right)\left(I+A_{n}\right)
\end{aligned}
$$

where $\left\{\boldsymbol{A}_{\boldsymbol{n}}, \boldsymbol{n} \in \mathbb{N}\right\} \subset \boldsymbol{L}(\boldsymbol{\Omega}, \boldsymbol{X} ; \boldsymbol{X})$ is a sequence of independent random linear operators and $\mathrm{I}$ is a unit operator.

In this paper, we introduce and establish limit theorems for sequences of martingales of random bounded linear operators. As an application, the infinite product of martingale differences of random operators taking values in a separable Banach space is investigated.

\section{Preliminaries and some useful lemmas}

Let $\mathrm{X}$ be a real separable Banach space with norm $\|$.$\| and (\Omega, \mathcal{F}, P)$ be a complete probability space. A measurable mapping $\xi$ from $(\Omega, \mathcal{F})$ into $(\mathrm{X} ; \mathcal{B}(\mathrm{X}))$ is called an X-valued random variable. The set of all $\mathrm{X}$-valued random variables is denoted by $\mathrm{L}_{0}^{\mathrm{X}}(\Omega)$. We do not distinguish two X-random variables which are equal almost surely. The space $\mathrm{L}_{0}^{\mathrm{X}}(\Omega)$ is equipped with the topology of convergence in probability. If a sequence $\left\{\xi_{n}, \mathrm{n} \geq 1\right\}$ of $\mathrm{L}_{0}^{\mathrm{X}}(\Omega)$ converges to $\xi$ in probability then we write $\mathrm{p}-\lim _{\mathrm{n} \rightarrow \infty} \xi_{\mathrm{n}}=\xi$, it is said that $\left\{\xi_{\mathrm{n}}, \mathrm{n} \geq 1\right\}$ converges to $\xi$ in $\mathrm{L}_{0}^{\mathrm{X}}(\Omega)$. The set of all $\mathrm{X}$-valued random variables $\xi$ which satisfy $\mathrm{E}\|\xi\|^{\mathrm{p}}<\infty$ is denoted by $\mathrm{L}_{\mathrm{p}}^{\mathrm{X}}(\Omega)$. We know that $\mathrm{L}_{\mathrm{p}}^{\mathrm{X}}(\Omega)(\mathrm{p} \geq 1)$ with norm $\|\xi\|_{L_{p}}=\left(E\|\xi\|^{p}\right)^{1 / p}$ is a Banach space.

Definition 2.1. ([10]) In a Banach space $X$ with Radon-Nikodym property, if every X-valued $\sigma$ additive set-funtion $\mu$ of bounded variation (that is, $V_{\mu}(\Omega)$ is finite) which is absolutely continuous with respect to $P$ has an intergral resresentation, that is exist $f \in L_{1}^{X}(\Omega)$ such that $\mu(A)=$ $\int_{A} f(s) P(d(s))$ for all $A \in \mathcal{F}$.

Theorem 2.2. ([10]) The Banach space $X$ and a probability space $(\Omega, \mathcal{F}, P)$ the following statement are equivalent when holding for all $\mathrm{X}$-valued martingales $\left\{\xi_{n}, \mathcal{F}_{n}, n \geq 1\right\}$.

1. If $\sup _{n} E\left\|\xi_{n}\right\|<\infty$ then $\lim _{n \rightarrow \infty} \xi_{n}=\xi_{\text {exists a.s. }}$ 
2. If $\sup _{n} E\left\|\xi_{n}\right\|^{p}<\infty(1<p<\infty)$ then exists $\quad \xi \in L_{p}^{Y}(\Omega)$ with $\lim _{n \rightarrow \infty} E\left\|\xi_{n}-\xi\right\|^{p}=0$.

3. The space $\mathrm{X}$ has the $\mathrm{R}-\mathrm{N}$ with respect to $(\Omega, \mathcal{F}, P)$.

Definition 2.3. ([3]) Let $\mathrm{X}, \mathrm{Y}$ be separable Banach space. A linear continuous mapping A from $\mathrm{X}$ into $L_{0}^{Y}(\Omega)$ is said to be a random linear operator from $X$ into $Y$.

Definition 2.4. Let $X, Y$ be real separable Banach spaces, $A, A_{n}(n \geq 1)$ be random linear operators from $\mathrm{X}$ into $\mathrm{Y}$.

1. $A_{n}$ is said to converge almost surely to $A$ and we write $A_{n} \rightarrow A$ as $n \rightarrow \infty$ if $A_{n}(x) \rightarrow A(x)$ a.s for all $x \in X$.

2. $A_{n}$ is said to converge to $A$ in mean of order $p$ (or in $L_{p}$ for short) as $n \rightarrow \infty(p>0)$ and we write $A_{n} \rightarrow A$ in $L_{p}$ as $n \rightarrow \infty$ if $\lim _{n \rightarrow \infty} E\left\|A_{n}(x)-A(x)\right\|^{p}=0$ for all $x \in X$.

Definition 2.5. ([3]) A random linear operator $A$ from $X$ into $Y$ is said to be bounded if there exists a real-valued random variable $\mathrm{k}(\omega)$ such that for each $\mathrm{x} \in \mathrm{X},\|\operatorname{Ax}(\omega)\| \leq \mathrm{k}(\omega)\|\mathrm{x}\|$ a.s.

By Theorem 3.1 in [3], there exists a mapping $T_{A}: \Omega \rightarrow L(X, Y)$ such that

$$
A x(\omega)=T_{A}(\omega) x \text { a.s. }
$$

It is easy to see that $T_{A}$ is unique, i.e., if $T_{A}^{(1)}, T_{A}^{(2)}$ satisfy (1) then

$T_{A}^{(1)}(\omega)=T_{A}^{(2)}(\omega)$ a.s.

Let $\mathrm{A}$ be a random bounded linear operator from a separable Banach space $\mathrm{X}$ into a separable a Banach space $\mathrm{Y}$. [3] defined the extension of A, which is a linear continuous mapping $\tilde{A}$ from $L_{0}^{X}(\Omega)$ in to $L_{0}^{X}(\Omega)$ by the following method.

- If $u$ is a $X$ - valued simple random variable, $u(\omega)=\sum_{i=1}^{n} 1_{E_{i}} x_{i}$, then $\tilde{A} u=\sum_{i=1}^{n} 1_{E_{i}} A x_{i}$.

- If $u \in L_{0}^{X}(\Omega)$, let a sequence $\left\{u_{n}, n \geq 1\right\}$ of X-valued simple random variables and $p-$ $\lim _{n \rightarrow \infty} u_{n}=u$ then there exists $p-\lim _{n \rightarrow \infty} \tilde{A} u_{n}$ and the limit does not depend on the choice of the approximate sequence $\left\{u_{n}, n \geq 1\right\}$ and is denoted by $\tilde{A} u$.

From now on, for the sake of simplicity, we write $A u$ instead of $\tilde{A} u$. $A u$ is called the action of A on the $\mathrm{X}$-valued random variable $\mathrm{u}$.

Lemma 2.6. Let A be a random bounded linear operator, $A x(\omega)=T(\omega) x$ a.s. Then $u \in L_{0}^{E}(\Omega)$, $A u(\omega)=T(\omega)(u(\omega))$.

Proof. $\mathrm{By} \operatorname{Ax}(\omega)=\mathrm{T}(\omega)$ x a.s. then $\mathrm{x} \in \mathrm{E}$, there exist $\mathrm{D}_{\mathrm{x}}$ with $\mathrm{P}\left(\mathrm{D}_{\mathrm{x}}\right)=1$, such that

$\operatorname{Ax}(\omega)=\mathrm{T}(\omega) \mathrm{x}$ for all $\omega \in \mathrm{D}_{\mathrm{x}}$.

If $u$ is an $\mathrm{X}$-valued simple random variable, $\mathrm{u}=\sum_{i=1}^{n} 1_{E_{i}} x_{i}, E_{i} \in S$ then for all $\omega \in D=\bigcap_{i=1}^{n} D_{x_{i}}$, $P(D)=1$, we have

$$
A u(\omega)=\sum_{i=1}^{n} 1_{E_{i}} A x_{i}(\omega)=\sum_{i=1}^{n} 1_{E_{i}} T(\omega) x_{i}=T(\omega) u(\omega)
$$

If $u \in L_{0}^{E}(\Omega)$, let $u_{n}(n \geq 1)$ be a sequence of $X$-valued simple random variables,

$p-\lim _{n \rightarrow \infty} u_{n}=u$, we have $A u_{n}(\omega)=T(\omega)\left(u_{n}(\omega)\right)$ for all $\omega \in D_{n}, P\left(D_{n}\right)=0$ then $\omega \in D=$ $\bigcap_{i=1}^{n} D_{x_{i}}, P(D)=1, A u_{n}(\omega)=T(\omega)\left(u_{n}(\omega)\right)$.

For each $\epsilon>0$, we have 
Let $n \rightarrow \infty$ and $r \rightarrow 0$, we obtain

$$
\begin{aligned}
P\left(\left\|T\left(u_{n}\right)-T(u)\right\|>\epsilon\right) & \leq P\left(\|T\|\left\|u_{n}-u\right\|>\epsilon\right) \\
& =P(\|T\| \geq \epsilon / r)+P\left(\left\|u_{n}-u\right\| \geq r\right)
\end{aligned}
$$

$\lim _{n \rightarrow \infty} P\left(\left\|T\left(u_{n}\right)-T(u)\right\|>\epsilon\right)=0$ this implies $p-\lim _{n \rightarrow \infty} T\left(u_{n}\right)=T(u)$.

In (2), let $n \rightarrow \infty$, we have

$$
A u(\omega)=T(\omega)(u(\omega)) \text { a.s. }
$$

\section{Lemma 2.7.}

Let $\mathrm{B}$ be a random bounded linear operator from a separable Banach space $\mathrm{X}$ into a separable Banach space $\mathrm{Y}, B x=T_{B} x$ a.s. for each $x \in X, \mathcal{G}$ be a sub- $\sigma$-algebra of $\mathcal{F}$. Then for each $\epsilon>0$, we have

$P(E\|B u\| \mid \mathcal{G})>\epsilon \leq P\left(E\left(\left\|T_{B}\right\|\|u\| \mid \mathcal{G}\right)>\epsilon / r\right)+P(\|u\|>r)$.

Proof. By Lemma 2.6, for each $u \in L_{0}^{X}(\Omega), \operatorname{Bu}(\omega)=T_{B}(\omega) u(\omega)$, so we have

$$
\begin{aligned}
& P(E\|B u\| \mid \mathcal{G})=P(E(\|\tau u\| \mid \mathcal{G})>\epsilon) \\
& \leq P\left(E\left(\left\|T_{B}\right\|\|u\| \mid \mathcal{G}\right)>\epsilon,\|u\|<r\right)+P(\|u\|>r) \\
& \leq P\left(E\left(\left\|T_{B}\right\|\|u\| \mid \mathcal{G}\right)>\epsilon / r\right)+P(\|u\|>r) .
\end{aligned}
$$

\section{Lemma 2.8 .}

Let $\mathrm{A}$ be a random bounded linear operator, $\mathcal{G}$ be a sub- $\sigma$-algebra of $\mathcal{F}$. Suppose that $\mathrm{E}(A x \mid \mathcal{G})=$ 0 for all $x \in X$. Then for each $u \in \mathcal{G}$, we have $\mathrm{E}(A u \mid \mathcal{G})=0$.

Proof. If $u$ is an $X$-valued simple random variable $u=\sum_{i=1}^{n} 1_{E_{i}} x_{i}$ then $\tilde{A} u=\sum_{i=1}^{n} 1_{E_{i}} A x_{i}$, so

$E(A u \mid g)=\sum_{i=1}^{n} E\left(1_{E_{i}} A x_{i} \mid \mathcal{G}\right)=\sum_{i=1}^{n} 1_{E_{i}} E\left(A x_{i} \mid \mathcal{G}\right)=0$.

If $u \in L_{0}^{X}(\Omega)$, there exists a sequence $\left\{u_{n}, n \geq 1\right\}$ of $X$-valued simple random variables such that $p-\lim _{n \rightarrow \infty} u_{n}=u$. Using Lemma 2.7, $E\left(A u_{n} \mid \mathcal{G}\right)$ converges to $E(A u \mid \mathcal{G})$ in $L_{0}^{X}(\Omega)$. Hence $E(A u \mid \mathcal{G})=p-\lim _{n \rightarrow \infty} E\left(A u_{n} \mid \mathcal{G}\right)=0$.

Definition 2.9. Let $A$ be a random bounded linear operator from a separable Banach space $X$ into a separable Banach space $\mathrm{Y}$ and $\mathcal{F}(\mathrm{A})$ denotes the $\sigma$-algebra generated by the family $\{\mathrm{Ax}, \mathrm{x} \in \mathrm{X}\}$.

Set $\mathcal{F}_{\mathrm{n}}=\sigma\left(\mathcal{F}\left(\mathrm{A}_{\mathrm{i}}\right), \mathrm{i} \leq \mathrm{n}\right)$. The random bounded operators $\left\{\mathrm{A}_{\mathrm{n}}, \mathrm{n} \geq 1\right\}$ are said to be martingale sequence of random bounded linear operations if $E\left(A_{n+1} x \mid \mathcal{F}_{n}\right)=A_{n} x$ for all $x \in X, n \geq 1$.

\section{Main results}

Let $\left\{A_{n}, n \geq 1\right\}$ be a sequence martingale of bounded random operators from $\mathrm{X}$ into $\mathrm{X}$. There exist mappings $T_{n}: \Omega \rightarrow L(X, X)$ such that

$$
A_{n} x(\omega)=T_{n}(\omega) x \text { a.s. }
$$

We have following theorem.

Theorem 3.1. Suppose that $\mathrm{X}$ has the Radon-Nikodym (R-N) property, let $p \geq 1,\left\{A_{n}, n \geq 1\right\}$ be a sequence martingale of random bounded linear operators from $\mathrm{X}$ into $\mathrm{X}$, then

1. $\left\|T_{n}(\omega)\right\|(n \in N)$ are real-valued random variables. 
2. If

$$
\sup _{n \geq 1} E\left\|T_{n}\right\|<\infty
$$

then there exists a random bounded linear operator $\mathrm{A}$ such that the sequence $\left\{A_{n}, n \geq 1\right\}$ converges a.s. to A. Moreover, $\left\|T_{n}\right\|$ converges a.s.

3. If

$$
\sup _{n \geq 1} E\left\|T_{n}\right\|^{p}<\infty, p>1
$$

then there exists a random bounded linear operator $\mathrm{A}$ such that the sequence $\left\{A_{n}, n \geq 1\right\}$ converges in $L_{p}$ to $\mathrm{A}$.

Proof. 1. For each $k \in N$, let $\left\{x_{n}, n \geq 1\right\}$ be a sequence dense in the unit ball $\{x \in X:\|x\|=$ $1\}$ then for all $\omega \in \Omega$,

$$
\left\|T_{k}(\omega)\right\|=\sup _{n \geq 1}\left\|T_{k}(\omega) x_{n}\right\| .
$$

Since

$$
A_{k} x(\omega)=T_{k}(\omega) x \text { a.s }
$$

there exist a set $\mathrm{D}$ of probability one such that for each $\omega \in D$, $A_{k} x_{n}(\omega)=T_{k}(\omega) x_{n}$ for all $n \in N$.

Then fix $\omega \in D$, we have

$$
\left\|T_{k}(\omega)\right\|=\sup _{n \geq 1}\left\|T_{k}(\omega) x_{n}\right\|=\sup _{n \geq 1}\left\|A_{k}(\omega) x_{n}\right\| .
$$

So $\left\|T_{A_{k}}\right\|(k \in N)$ are random variables.

For each $x \in X$, we have $\mathrm{E}\left\|A_{n} x\right\| \leq E\left\|T_{n}\right\|\|x\|$ then

$$
\sup _{n \geq 1} \mathrm{E}\left\|A_{n} x\right\| \leq\|x\| \sup _{n \geq 1} E\left\|T_{n}\right\|<\infty
$$

so there exists $A x \in L_{1}^{X}(\Omega), A_{\boldsymbol{n}} \boldsymbol{x} \rightarrow A x$ a.s. Moreover,

$$
\sup _{n \geq 1} P\left(\left\|T_{n} \omega\right\|>\epsilon\right) \leq \frac{\sup _{n \geq 1} E\left\|T_{n}\right\|}{\epsilon} \rightarrow 0 \text { as } \epsilon \rightarrow 0
$$

then $\left\{\left\|T_{n}\right\|, n \geq 1\right\}$ is bounded in probability. By Theorem 5.4 [5], $A x$ is random bounded linear operator.

Next, for each $n \geq 1$ and for all $\epsilon>0$ then there exists an element $a$ in the unit ball, such that

$$
\left\|T_{n}\right\|-\epsilon \leq\left\|T_{n} a\right\|=\left\|A_{n} x\right\|=\left\|E\left(A_{n+1} a \mid \mathcal{F}_{n}\right)\right\| \leq E\left(\left\|A_{n+1} a\right\| \mid \mathcal{F}_{n}\right) \leq E\left(\left\|T_{n+1}\right\| \mid \mathcal{F}_{n}\right) .
$$

Let $\epsilon \rightarrow 0$ then

$\left\|T_{n}\right\| \leq E\left(\left\|T_{n+1}\right\| \mid \mathcal{F}_{n}\right)$ for all $n \geq 1$,

so $\left\{\left\|T_{n}(\omega)\right\|, n \in N\right\}$ is a real-valued sub martingale. Since $\sup _{n \geq 1} E\left\|T_{n}\right\|<\infty$ then $\left\|T_{n}\right\|$ converges a.s.

For each $x \in X$, we have

$$
\mathrm{E}\left\|A_{n} x\right\|^{p} \leq E\left\|T_{n}\right\|^{p}\|x\|^{p} \text { then } \sup _{n \geq 1} \mathrm{E}\left\|A_{n} x\right\|^{p} \leq\|x\|^{p} \sup _{n \geq 1} E\left\|T_{n}\right\|^{p}<\infty
$$

so there exists $A x \in L_{\boldsymbol{p}}^{X}(\Omega), A_{n} x \rightarrow A x$ in $L_{p}$. Moreover, 
$\sup _{n \geq 1} P\left(\left\|T_{n} \omega\right\|>\epsilon\right) \leq \frac{\sup _{n \geq 1} E\left\|T_{n}\right\|}{\epsilon} \rightarrow 0$ as $\epsilon \rightarrow 0$.

Therefore, $\left\{\left\|T_{n}\right\|, n \geq 1\right\}$ is bounded in probability. By Theorem 5.4 [3], Ax is a random bounded linear operator.

Theorem 3.2. Suppose that $\mathrm{X}$ has the Radon-Nikodym (R-N) property, let $p \geq 1,\left\{A_{n}, n \geq 1\right\}$ be a sequence martingale of random bounded linear operators from $\mathrm{X}$ into $\mathrm{X}$, then

1 . If

$$
\sup _{n \geq 1} E\left\|T_{n}\right\|<\infty
$$

Then there exists a random bounded linear operator $\mathrm{A}$ such that the sequence $\left\{A_{n} u, n \geq 1\right\}$ converges a.s. to $A u$ for all $u \in L_{0}^{E}\left(\Omega, \mathcal{F}_{1}\right)$.

2. If

$$
\sup _{n \geq 1} E\left\|T_{n}\right\|^{p}<\infty(p>1)
$$

then there exists a random bounded linear operator $\mathrm{A}$ such that the sequence $\left\{A_{n} u, n \geq 1\right\}$ converges a.s. to $A u$ for all $u \in L_{q}^{E}\left(\Omega, \mathcal{F}_{1}\right)$ where $\frac{1}{p}+\frac{1}{q}=1$.

3. If

$$
\sup _{n \geq 1} E\left\|T_{n}\right\|^{q}<\infty(q>1)
$$

then there exists a random bounded linear operator $A$ such that the sequence $\left\{A_{n}, n \geq 1\right\}$ converges in $\mathrm{L}_{\mathrm{r}}(\mathrm{q}>\mathrm{r}>1)$ to $\mathrm{Au}$ for all $\mathrm{u} \in \mathrm{L}_{\mathrm{p}}^{\mathrm{E}}\left(\Omega, \mathcal{F}_{1}\right)$ where $\frac{\mathrm{r}}{\mathrm{p}}+\frac{\mathrm{r}}{\mathrm{q}}=1$.

Proof. 1. By Theorem 3.1, then exists a random bounded linear operator $A$ such that the sequence $\left\{A_{n}, n \geq 1\right\}$ converges a.s. to $A$. Moreover, $\sup _{n \geq 1}\left\|T_{n}\right\|<\infty$ a.s.

Let $u(\omega)=\sum_{i=1}^{n} 1_{E_{i}} x_{i}$ be a simple random variable, by $A_{n} x_{i} \rightarrow A x_{i}$ a.s. as $n \rightarrow \infty$, then

$$
A_{n} u=\sum_{i=1}^{n} 1_{E_{i}} A_{n} x_{i} \rightarrow \sum_{i=1}^{n} 1_{E_{i}} A x_{i}=A u \text { a.s }
$$

If $u \in L_{0}^{E}(\Omega)$, for each $t>0, \epsilon>0$. By $\sup _{n \geq 1}\left\|T_{n}\right\|<\infty$ a.s. then there exist $r>0$ such that

$$
P\left(\sup _{n \geq 1}\left\|T_{n}-T\right\| \geq t / 2 r\right)<\epsilon / 3 .
$$

Let $u_{0}$ be a simple random variable and

$$
P\left(\left\|u-u_{0}\right\| \geq r\right)<\epsilon / 3 .
$$

Moreover, by (3) there exists $\mathrm{N}$, such that for all $n \geq N$,

$$
P\left(\sup _{i \geq n}\left\|A_{i} u_{0}-A u_{0}\right\| \geq t / 2 r\right)<\epsilon / 3 .
$$

For each $n \geq N$, 


$$
\begin{gathered}
P\left(\sup _{i \geq n}\left\|A_{i} u-A u\right\| \geq t\right) \\
\leq P\left(\sup _{i \geq n}\left\|\left(A_{i}-A\right)\left(u_{0}-u\right)\right\| \geq t / 2\right)+P\left(\sup _{i \geq n}\left\|\left(A_{i}-A\right) u_{0}\right\| \geq t / 2\right) \\
\leq P\left(\sup _{i \geq n}\left\|\mathrm{~T}_{\mathrm{i}}\right\|\left\|\left(u_{0}-u\right)\right\| \geq t / 2\right)+P\left(\sup _{i \geq n}\left\|B_{i} u_{0}\right\| \geq t / 2\right) \\
\leq P\left(\sup _{i \geq n} \mathrm{~T}_{\mathrm{n}}-\mathrm{T} \| \geq t / 2 r\right)+P\left(\sup _{i \geq n}\left\|u-u_{0}\right\| \geq r\right)+P\left(\sup _{i \geq n}\left\|\left(A_{i} u_{0}-A u_{0}\right)\right\| \geq t / 2\right) \\
\leq \epsilon / 3+\epsilon / 3+\epsilon / 3=\epsilon
\end{gathered}
$$

Consequently, $\lim _{n \rightarrow \infty} A_{n} u=A u$ a.s.

2. By Lemma 2.8, for all $u \in L_{p}^{E}\left(\Omega, \mathcal{F}_{1}\right)$ then $\left\{A_{n} u, n \geq 1\right\}$ is a martingale sequence, using Lemma 2.6, we obtain

$$
E\left\|A_{n} u\right\|=E\left\|T_{n} u\right\| \leq E\left(\left\|T_{n} u\right\|^{p}\right)^{1 / p} E\left(\left\|T_{n} u\right\|^{q}\right)^{1 / q}
$$

and

$$
\sup _{n \geq 1} E\left\|A_{n} u\right\|^{r} \leq \sup _{n \geq 1} E\left(\left\|T_{n} u\right\|^{p}\right)^{1 / p} E\left(\left\|T_{n} u\right\|^{q}\right)^{1 / q}<\infty .
$$

But $\mathrm{X}$ has the Radon-Nikodym (R-N) property, then $A_{n} u \rightarrow A u$ a.s.

3. $E\left\|A_{n} u\right\|^{r}=E\left\|T_{n} u\right\| \leq E\left(\left\|T_{n} u\right\|^{p}\right)^{1 / p} E\left(\left\|T_{n} u\right\|^{q}\right)^{1 / q}$. We have

$$
\sup _{n \geq 1} E\left\|A_{n} u\right\|^{r} \leq \sup _{n \geq 1} E\left(\left\|T_{n} u\right\|^{p}\right)^{1 / p} E\left(\left\|T_{n} u\right\|^{q}\right)^{1 / q}<\infty .
$$

Since $\mathrm{X}$ has the Radon-Nikodym property, then $A_{n} u \rightarrow A u$ in $L_{r}$

Let $\left\{A_{n}, n \geq 1\right\}$ be a martingale sequence of random bounded linear operators from $\mathrm{X}$ into $\mathrm{X}$. We set $B_{n} x=A_{n} x-A_{n-1} x$, then we have $E\left(B_{n} x \mid \mathcal{F}_{n-1}\right)=0$ and $B_{n} x=A_{n} x-A_{n-1} x=\left(T_{n}-\right.$ $\left.T_{n-1}\right) x:=T_{B_{n}} x$, we said $\left\{B_{n}, n \geq 1\right\}$ is a martingale difference of random bounded linear operators.

Define the sequence $\left\{A_{n}^{b}, n \geq 1\right\}$ and $\left\{A_{n}^{f}, n \geq 1\right\}$ by

$$
\begin{gathered}
A_{n}^{b}=\left(I+B_{n}\right)\left(I+B_{n-1}\right) \ldots\left(I+B_{1}\right), \\
A_{n}^{f}=\left(I+B_{1}\right) \ldots\left(I+B_{n-1}\right)\left(I+B_{n}\right) .
\end{gathered}
$$

The problem is to study the convergence of the sequence $\left\{A_{n}^{b}, n \geq 1\right\}$ and $\left\{A_{n}^{f}, n \geq 1\right\}$ i.e. the convergence of the products

$$
\prod_{k=\infty}^{1}\left(I+B_{k}\right) \text { and } \quad \prod_{k=1}^{\infty}\left(I+B_{k}\right) .
$$

Theorem 3.3. Suppose that $X$ has the Radon-Nikodym (R-N) property, let $p \geq 1,\left\{B_{n}, n \geq 1\right\}$ be a martingale difference sequence of random bounded linear operators from $\mathrm{X}$ into $\mathrm{X}$,

1. If

$$
E \prod_{n=1}^{\infty}\left\|I+T_{B_{n}}\right\|<\infty
$$

then the product $\prod_{k=\infty}^{1}\left(I+B_{k}\right)$ and the product $\prod_{k=1}^{\infty}\left(I+B_{k}\right)$ converge a.s.

2. If 


$$
E \prod_{n=1}^{\infty}\left\|I+T_{B_{n}}\right\|<\infty
$$

then the product $\prod_{k=\infty}^{1}\left(I+B_{k}\right)$ and the product $\prod_{k=1}^{\infty}\left(I+B_{k}\right)$ converge in mean of order $\mathrm{p}$.

Proof.

1. We have

then

$$
V_{n+1} x=U_{n} x+B_{n+1}\left(U_{n} x\right)
$$

$$
E\left(U_{n+1} x \mid \mathcal{F}_{n}\right)=U_{n} x+E\left(B_{n+1}\left(U_{n} x\right) \mid \mathcal{F}_{n}\right) .
$$

Put $x=U_{n} x$, so $u \in \mathcal{F}_{n}, E\left(B_{n+1}(x) \mid \mathcal{F}_{n}\right)=0$ for all $x \in E$. By Lemma 2.8, we obtain $E\left(B_{n+1}\left(U_{n} x\right) \mid \mathcal{F}_{n}\right)=0$,

so we have $E\left(U_{n+1} x \mid \mathcal{F}_{n}\right)=0$ or $\left\{U_{n+1} x ; \mathcal{F}_{n}\right\}$ is a martingale sequence.

Moreover,

$$
E\left\|U_{n} x\right\|=E\left\|\prod_{k=1}^{n}\left(I+T_{k}\right) x\right\| \leq E \prod_{k=1}^{n}\left\|\left(I+T_{k}\right)\right\|<\infty .
$$

This implies $\left\{U_{n} x, n \geq 1\right\}$ is convergent a.s.

The proof of 2) is the same as that of 1).

\section{Acknowledgements}

This research has been supported by Vietnam National University, Hanoi (grant no. QG.16.09)

\section{References}

[1] D.H. Thang, T.N. Anh, On random equations and applications to random fixed point theorems, Random Oper.Stoch.Equ. 18(2010), 199-212.

[2] D.H. Thang, T.C. Son, On the convergence of the product of independent random operators, Stochas.Int. J. Prob. Stochas. Process. 88(2016), 927-945.

[3] D.H. Thang and N. Thinh, Random bounded operators and their extension, Kyushu J.Math. 58 (2004), 257-276.

[4] D.H. Thang, N. Thinh, Generalized random linear operators on a Hilbert space, Stochas. Int. J. Prob. Stochas. Process. 85(2013), 1040-1059.

[5] Dung, L.V., Son, T. C. and Tien, N. D., L1 bounds for some martingale central limit theorems, Lithuanian Mathematical Journal, 54 (1), 48-60 (2014).

[6] T.C.Son and D.H. Thang, The Brunk-Prokhorov strong law of large numbers for fields of martingale differences taking values in a Banach space, Statistics \& Probability Letters (2013) 83: 1901-1910.

[7] T.C. Son and D.H. Thang, On the convergence of series of martingale differences with multidimensional indices, Journal of the Korean Mathematical Society, 52(5) (2015): 1023-1036.

[8] T.C.Son, D.H.Thang and L.V.Dung, Rate of complete convergence for maximums of moving average sums of martingale difference fields in Banach spaces, Statist.Probab.Lett. 82(2012), 1978-1985.

[9] Y. Kifer, Ergodic Theory of Random transformation, Birkhauser, (1986)

[10] S.D.Chatterji, Martingale convergence and the Radon-Nikodym theorem in Banach spaces, Math. Scand, 22 (1986) 21-41. 